\title{
Fetal Anemia Caused by Prenatal Gastrointestinal Bleeding: A Case Report
}

\author{
Kensuke Matsumoto1*, Masayuki Yamaguchi1, Taro Nonaka1, \\ Takayuki Enomoto', Kouichi Takakuwa² \\ ${ }^{1}$ Department of Obstetrics and Gynecology, Niigata University Graduate School of Medical \\ and Dental Sciences, Niigata, Japan \\ ${ }^{2}$ General Center for Perinatal, Maternal and Neonatal Medicine, Niigata University \\ General Hospital of Medical and Dental Sciences, Niigata, Japan \\ Email: "kensuke19821101@yahoo.co.jp
}

Received 16 March 2016; accepted 24 May 2016; published 27 May 2016

Copyright (C) 2016 by authors and Scientific Research Publishing Inc.

This work is licensed under the Creative Commons Attribution International License (CC BY). http://creativecommons.org/licenses/by/4.0/

(c) (i) Open Access

\section{Abstract}

A 39-year-old 34-week-pregnant woman was referred to our hospital for preterm labor and fetal dysfunction. Fetal middle cerebral artery systolic maximum blood flow velocity was high, so fetal anemia was suspected. Blood type was 0 -type $\mathrm{Rh}(+)$ and fetal hemoglobin was $0.4 \%$; tests for irregular antibody and human parvovirus B19 IgM were negative. A high brightness echoic mass was observed in fetal stomach bubble, and amniotic fluid appeared bright. Labor suppression was disabled, and emergency cesarean section was performed. Amniotic fluid turbidity was observed, and on suctioning the stomach content of the infant, turbid amniotic fluid containing blood was obtained. On placental pathological examination, the cause of bloody amniotic fluid was not identified. The infant's hemoglobin level was low at $8.7 \mathrm{~g} / \mathrm{dL}$, so the infant received red cell concentrate, with improvement of general condition.

\section{Keywords}

Fetal Anemia, MCA-PSV Is High, Prenatal Gastrointestinal Bleeding, Amniotic Fluid Brightness

\section{Introduction}

With the development of ultrasonic examination, it has become possible to diagnose the fetal anemia. Fetal anemia progresses to fetal hydrops and fetal dysfunction, and in the worst case, leads to intrauterine fetal death. For that reason, it is very important to search the cause of fetal anemia. Blood type incompatibility, fetomaternal transfusion

\footnotetext{
"Corresponding author.
} 
syndrome and human parvovirus B19 infection can be identified by blood tests for blood type, HbF, and human parvovirus B19 IgM, but fetal gastrointestinal bleeding is difficult to diagnose. Herein, we report the first case of fetal anemia due to fetal gastrointestinal bleeding and describe the clinical management and diagnostic challenges.

\section{Case Report}

A 39-year-old woman, Gravida 4 Para $2 \times 2$ times cesarean section, was referred to Niigata University General Hospital of Medical and Dental Science for advanced prenatal care. She was healthy with normal blood tests and no remarkable medical history; her height was $156 \mathrm{~cm}$ and body weight was $58 \mathrm{~kg}$. For the current pregnancy, she was undergoing prenatal care at a physician's clinic. At 33 weeks 5 days of pregnancy, she was admitted because of a complaint of decrease in fetal movement. Cardiotocography (CTG) did not reveal acceleration but revealed uterine contractions every 6 - $8 \mathrm{~min}$. Fetal breathing movement was not recognized on ultrasonography, and her biophysical profile score (BPS) was 6 points; therefore, she was admitted and tocolysis by hydrochloric acid ritodrine infusion was initiated. After admission, the uterine contractions subsided, and BPS at 33 weeks 6 days of pregnancy was 8 points but decreased to 6 points at 34 weeks. Since there was a high possibility of rapid delivery, she was transferred to our hospital. CTG showed reassuring fetal status (RFS), and uterine contractions were not identified. Estimated fetal weight was $2155 \mathrm{~g}$ and high brightness echo was observed in the fetal stomach bubble (Figure 1). Fetal middle cerebral artery systolic maximum blood flow velocity (MCA-PSV) was $84.11 \mathrm{~cm} / \mathrm{sec}$ (Figure 2). MCA-PSV was over 1.55 multiples of the median. Fetal anemia was strongly suggested, but cardiothoracic area ratio (CTAR) was $30 \%$ and heart enlargement was not observed. We did not observe signs of fetal hydrops such as pleural effusion and ascites. Amniotic fluid pocket was $5.34 \mathrm{~cm}$ and it showed an increase in amniotic fluid brightness (Figure 3 ). The mother's blood type was O-type Rh $(+)$ and fetal hemoglobin $(\mathrm{HbF})$ was $0.4 \%$; tests for irregular antibody and human parvovirus B19 IgM were negative. After admission, MCA-PSV remained high, so we prepared for cordocentesis and fetal transfusion, but labor suppression was disabled at 35 weeks 3 days of pregnancy, and she underwent an emergency cesarean section. Intraoperatively, amniotic fluid turbidity was observed; instead of the usual yellow fluid, it was dark brown, suggestive of prior bleeding from the fetus. The patient delivered a female infant weighing $2408 \mathrm{~g}$, with Apgar scores of 4 and 8 points at 1 and 5 min, respectively, and the following umbilical cord blood results: $\mathrm{pH}=7.33$, $\mathrm{BE}=-5.5$. The infant was promptly referred to a pediatric doctor. On suctioning the stomach content of the infant, a large amount of turbid amniotic fluid containing blood was obtained, and the respiratory status improved by skin irritation and oxygen administration; the infant was then admitted to the neonatal intensive care unit. The infant was placed in an incubator in $30 \%$ oxygen; her $\mathrm{SpO}_{2}$ was $88 \%-94 \%$, heart rate was 180 beats $/$ min, blood pressure was $59 / 26 \mathrm{mmHg}$, respiratory rate was $80-100$ times $/ \mathrm{min}$, and she expectorated mucus containing brown colored blood. Hemoglobin level was $8.7 \mathrm{~g} / \mathrm{dL}$, and after transfusion of red cell concentrate $60 \mathrm{~mL}$, her hemoglobin level improved to $13.0 \mathrm{~g} / \mathrm{dL}$. Her blood type was type-A Rh (+), irregular antibody was absent, and coagulation properties were normal. Intestinal malrotation was not detected on abdominal radiography, and

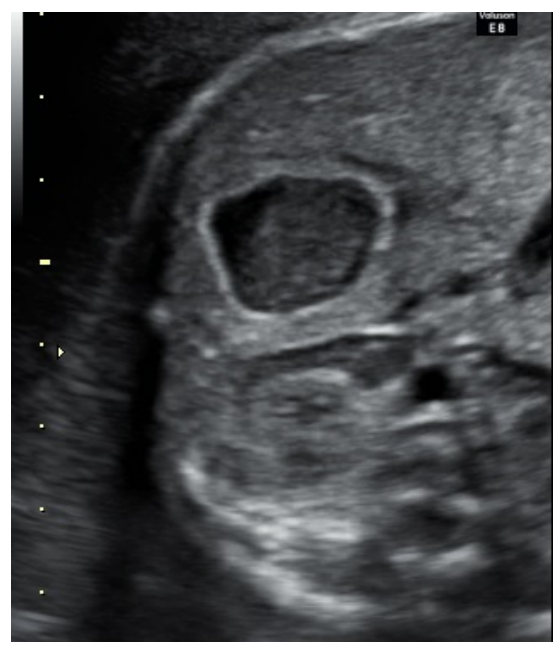

Figure 1. High brightness echo was observed in the fetal stomach bubble. 


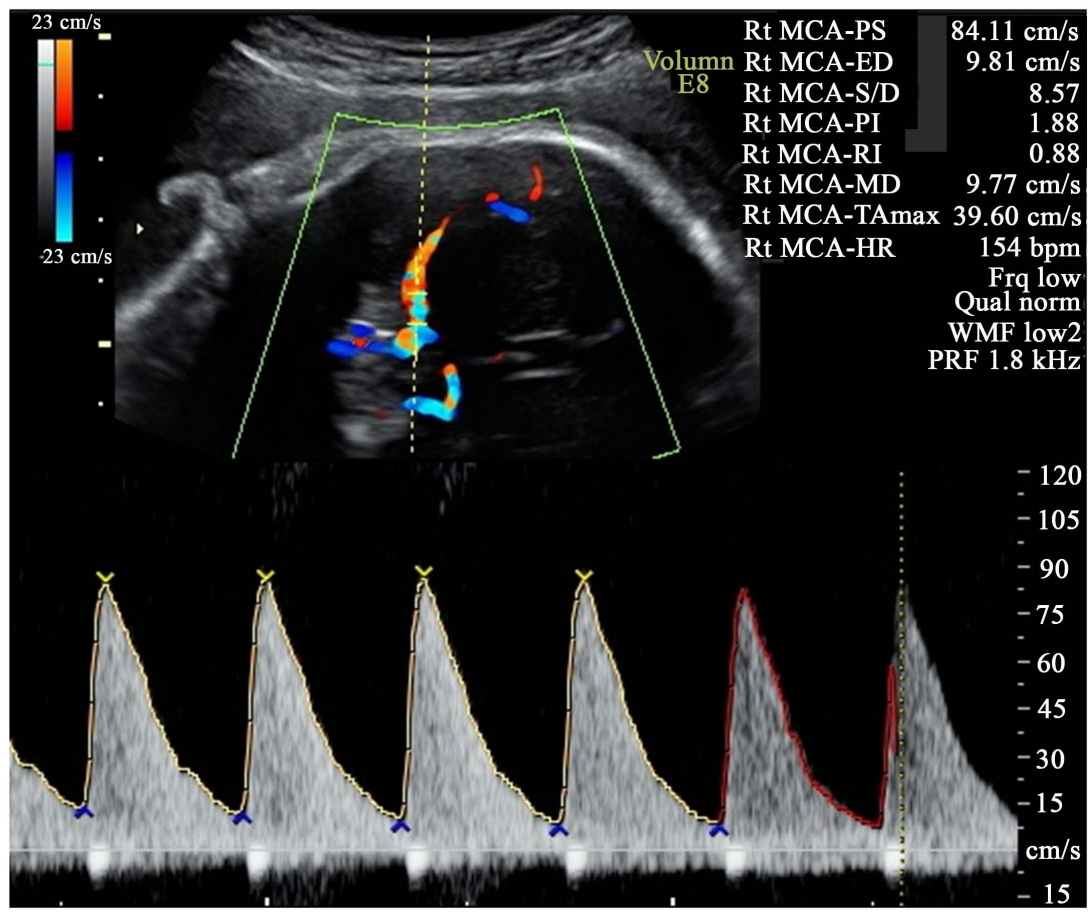

Figure 2. Fetal middle cerebral artery systolic maximum blood flow velocity (MCA-PSV) was high.

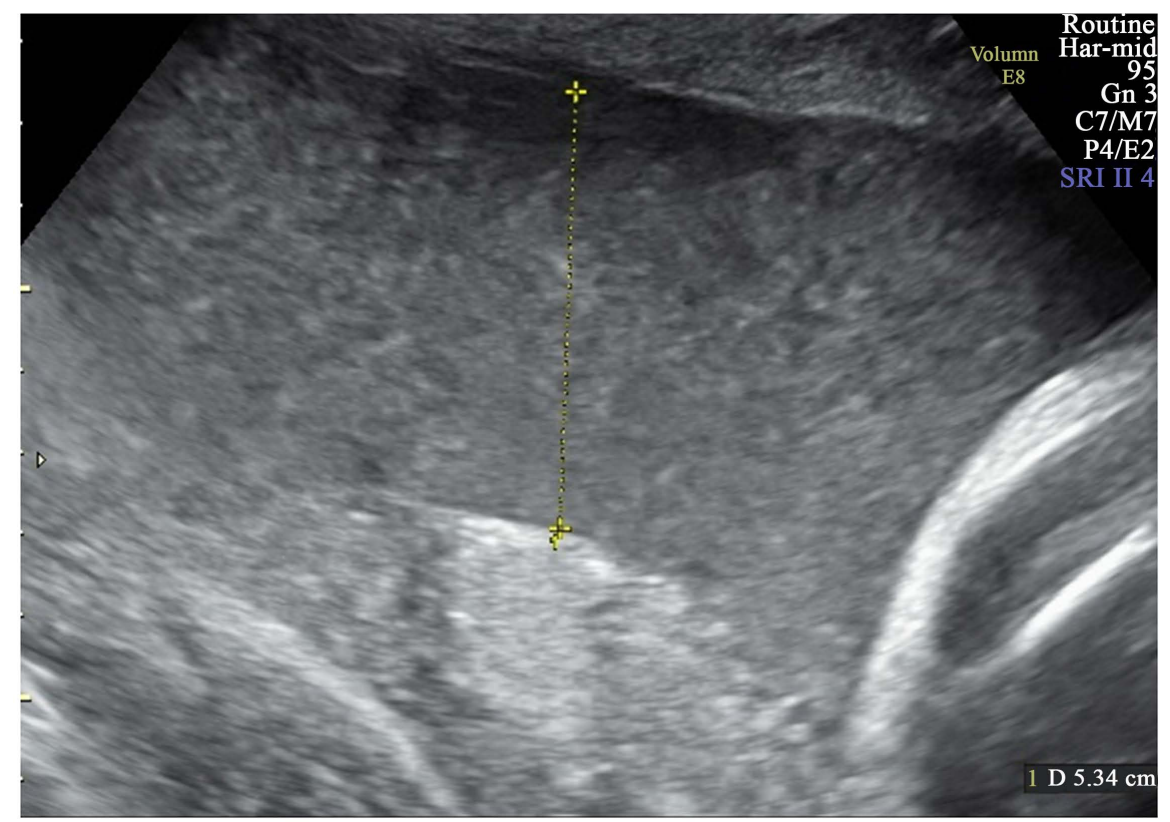

Figure 3. It showed an increase in amniotic fluid brightness.

gastrointestinal tumor was not detected on gastrointestinal contrast examination, but since the infant presented with blood-mingled vomiting and bloody stool, so gastrointestinal bleeding was suspected to be present from the prenatal stage. Her general condition gradually improved, and she was discharged day 43 . The placenta weighed $633 \mathrm{~g}$ and measured $24 \times 19 \times 2 \mathrm{~cm}$ in size, with no noteworthy findings. On pathological examination, only grade I chorioamnionitis was identified, but the cause of bloody amniotic fluid (placental hematoma or/and umbilical cord rupture) was not identified. 


\section{Discussion}

In recent years, ultrasonic pulse Doppler MCA-PSV measurement is said to be useful for identifying fetal anemia [1]-[5]. In this case, MCA-PSV was $\geq 1.55 \mathrm{MoM}$ (multiples of the median) advanced, so fetal anemia was suggested. The most common cause of fetal anemia is blood type incompatibility, and other causes include fetomaternal transfusion syndrome, human parvovirus B19 infection, congenital bleeding diathesis, and bleeding of the fetus [1] [4] [5]. In this case, blood type of the mother was O-type Rh (+), and that of the infant was A-type $\mathrm{Rh}(+)$, and fetal hemolytic anemia due to blood type incompatibility from irregular antibody was negative. In addition, $\mathrm{HbF}$ was $0.4 \%$, fetomaternal transfusion syndrome was unlikely, and human parvovirus B19 infection was also negative.

Amniotic fluid brightness is mainly caused by the vernix caseosa peeling off the fetus as it matures, but a few cases have reported meconium and bleeding as the causative factors [6]-[11]. The mother was 34 weeks pregnant, so vernix caseosa peeling off because of maturation of the fetus was unlikely. Color of the amniotic fluid was dark brown, and meconium was not recognized, possibly because of bleeding in the amniotic fluid. Although no obvious abnormality in the placenta and umbilical cord was identified, bloody stool and turbid amniotic fluid were suctioned from the infant's stomach, so the bleeding was assumed to have occurred from the fetus.

Fetal gastric bubble is usually visualized as a uniform body on low echoic image. If a high-brightness echo is observed in the fetal stomach bubble, it is a reservoir image of the decomposition products of swallowing the amniotic fluid [12]. This usually disappears in a few days. In this case, an emergency cesarean section was performed a few days after the mother was transferred to our hospital, so we were not able to follow the course in detail. Based on the stomach contents of the infant, we suspect that a hemorrhage was the cause of prenatal gastrointestinal bleeding.

\section{Conclusion}

Herein we report a case of fetal anemia caused by gastrointestinal bleeding. In cases exhibiting high MCA-PSV on ultrasonic inspection, high brightness echo of amniotic fluid, and high-brightness echo within the fetal stomach bubble, it is necessary to consider the possibility of fetal gastrointestinal bleeding.

\section{Conflict of Interest}

The authors have no conflicts of interest to declare.

\section{References}

[1] (2014) Japan Society of Obstetrics and Gynecology: Obstetrics and Gynecology Practice Guidelines Obstetrics Compilation.

[2] Oepkes, D., Seaward, P.G., Vandenbussche, F.P., et al. (2006) Doppler Ultrasonography versus Amniocentesis to Predict Fetal Anemia. The New England Journal of Medicine, 355, 156-164. http://dx.doi.org/10.1056/NEJMoa052855

[3] Mari, G., Deter, R.L., Carpenter, R.L., et al. (2000) Noninvasive Diagnosis by Doppler Ultrasonography of Fetal Anemia due to Maternal Red-Cell Alloimmunization. The New England Journal of Medicine, 342, 9-14. http://dx.doi.org/10.1056/NEJM200001063420102

[4] Sasaki, T., Niiro, E., Shigemitsu, A., Masaki, S., Yamaguchi, E., Mizuta, H. and Yamada, Y. (2013) A Case of Fetomaternal Transfusion Syndrome with Fetal Anemia Suspected on Doppler Ultrasonography. Advanced Obstetrics \& Gynecology, 65, 397-401.

[5] Japan Society of Perinatal and Neonatal Medicine (2012) Perinatal Care Workbook.

[6] Shibata, M., Betsumiya, S., Kawakita, T., Yonetani, N., Ushigoe, K., Myogo, K. and Ino, H. (2012) Echogenic Amniotic Fluid in Late-Term Pregnancy. Tokushima Red Cross Hospital Medical Journal, 17, 12-15.

[7] Ryo, E., Ikeya, M., Kasai, Y. and Sugimoto, M. (2002) Prenatal Upper Gastrointestinal Bleeding. Ultrasound in Obstetrics and Gynecology, 20, 308-310. http://dx.doi.org/10.1046/j.1469-0705.2002.00779_5.x

[8] Wen, H.H., Chen, M.H., Ho, M.M. and Hwang, K.C. (1992) Fetal Gastric Ulcer Presenting with Bloody Amniotic Fluid. Journal of Pediatric Gastroenterology and Nutrition, 15, 455-457. http://dx.doi.org/10.1097/00005176-199211000-00017

[9] Tam, G. and Al Dughaishi, T. (2013) Case Report and Literature Review of Very Echogenic Amniotic Fluid at Term 
and Its Clinical Significance. Oman Medical Journal, 28, 6. http://dx.doi.org/10.5001/omj.2013.129

[10] Mingen, E., Tutuncu, L. and Muhchu, M. (2005) Pregnancy Outcome in Women with Echogenic Amniotic Fluid at Term Gestation. International Journal of Gynecology and Obstetrics, 88, 314-315. http://dx.doi.org/10.1016/j.ijgo.2004.11.021

[11] Decoret, E., Sibony, O., Vuillard, E., Bedu, A., Faure, O., Mandelbrot, L., Mullor, F., Oury, J.F. and Blot, P.H. (1994) Prenatal Diagnosis of in Utero Fetal Gastrointestinal Bleeding. Fetal Diagnosis and Therapy, 9, 252-255. http://dx.doi.org/10.1159/000263942

[12] Karippaliyil, B., Kannan, M. and Karippaliyil, M. (2014) Fetal Gastric Pseudomass at 30 Weeks of Gestation and Its Regression after 17 Days of Birth. Indian Journal of Radiology and Imaging, 24, 160-162. http://dx.doi.org/10.4103/0971-3026.134403 